\title{
Childhood diabetes: a practical guide for child psychiatrists
}

\section{Anne Worrall-Davies}

Diabetes mellitus in childhood is almost always insulin-dependent diabetes mellitus (IDDM). The UK prevalence is 1 in 600 children under 16 years of age (Metcalfe \& Baum, 1991) and the incidence is 13.5 new cases per 100000 children per year aged 15 years and under. One quarter of newly diagnosed cases in the UK are under the age of five years, half are aged between 10 and 14 years.

\section{Paediatric diabetes teams}

Children with IDDM are generally looked after by a specialist paediatric diabetes team (Baum, 1990), which might comprise:

(a) a consultant paediatrician with an interest in IDDM;

(b) one or more diabetes nurse specialists;

(c) a paediatric dietician;

(d) a medical social worker;

(e) a child psychologist or child psychiatrist.

\section{Paediatric management}

Children usually present with polyuria, polydipsia, malaise and weight loss. They rarely present in diabetic coma. Post-diagnosis, the initial stabilisation may be carried out during a brief (24-hour) hospital stay or in the community at home with outreach support. Insulin administration is tailored to the individual child and depends on the child's age, weight and blood glucose value. This forms only part of the stabilisation programme.

Education of the child and family has to begin quickly even at this early stage. The concept of the multi-disciplinary team approach to care is explained and as many of the team as possible should aim to meet the family. Instruction is given about capillary blood glucose testing and recordkeeping, the broad concepts of a suitable dietary regime and management of the child in the event of hypoglycaemia. Deliberate induction of a hypoglycaemic episode, as practised in the past, plays no part in modern management of children with IDDM and should never be undertaken as it simply frightens children and parents.

Subsequent health care takes place predominantly in the community with three or four hospital outpatient appointments a year. The goal of management is to achieve relatively steady blood sugar levels in order to prevent both short-term morbidity (hypoglycaemic or hyperglycaemic ketotic episodes) and long-term microvascular complications. This is achieved by titrating insulin injections, diet and exercise in the framework of a healthy lifestyle. Current insulin regimes typically use twice-daily injections of pre-mixed short- and long-acting formulations in an 'insulin pen'. Injections are administered by the children when they feel ready to do so, and by their parents until that time. This may be as early as five or six years old, or as late as 13 or 14 years old, depending on the child.

\section{Diet}

Diet is based on good eating habits for all the family. Distribution of meals and snacks should aid the avoidance of hypoglycaemia or hyperglycaemia and should be based on a carbohydrate and/or fat exchange system of 'swaps'. A carbohydrate swap system might allow one item of food at snack time and two items at a mealtime to be taken from a list of carbohydrates, for example: pasta, rice, potato and milk (Magrath et $a l, 1993)$. A fat exchange system works similarly. 


\section{Blood sugar levels}

These are usually monitored at least daily, using capillary blood from a fingertip (fingerprick test) on a dipstick in a pre-calibrated glucose meter. Achieving blood sugar levels close to those found in healthy children is probably not advisable, since repeated hypoglycaemic episodes in children under seven years of age can lead to cognitive deficits (Diabetes Control and Complications Trial Research Group, 1993; Shield \& Baum, 1994).

\section{Psychological problems in children with IDDM}

Small-scale descriptive and analytic studies do not lead to a firm conclusion that emotional and behavioural problems are more common in children with diabetes than in healthy children (Court et al, 1988; Kovacs et al, 1990). However, the Ontario population study (Cadman et al, 1986) and a recent meta-analysis (Lavigne \& Faier-Routman, 1992) showed clearly that children with a chronic disease are twice as likely as healthy children to have a psychological problem. Overall, the prevalence of psychological problems in children with IDDM is 25-30\% higher than in healthy children, but this figure shows that most children with IDDM do not have psychological problems.

Of all children with IDDM, girls rather than boys report anxiety and depressive symptoms in the year after diagnosis, and the risk of developing psychological problems is greater the older the child. There is no recognised pattern of association between glycaemic control and psychological difficulties, although some studies suggest that poor control is associated with behaviour problems, and good control with emotional difficulties. The direction of effect is also not known, because most studies have been cross-sectional in design (Worrall, 1996). With the obvious exception of

Box 1. Services offered by child psychiatrists to a paediatric diabetes team

Assessment of urgent referrals within 24 hours Routine assessment and treatment

Role in preventive health care

Regular attendance at clinic or post-clinic discussion meetings

Staff support and training

Consultation with the paediatric team needle phobia, there are no specific problems to which children with IDDM are vulnerable.

Little is known about why a minority of children with IDDM develop psychological problems. Researchers in the field now recommend that it is more useful to think about the aetiology of psychological problems in children with chronic disease as a whole rather than in separate diagnostic groups. One good example of this is the 'stress and coping' model, which has been found to be useful in a range of paediatric chronic diseases (Wallander \& Varni, 1998).

\section{Range of services usefully offered by a child psychiatrist}

The list of services in Box 1 is not intended as a blueprint for a service to a paediatric diabetes team. However, one or more options might be appropriate at different stages in a child psychiatrist's involvement with the paediatric team and at different stages in the life-cycle of that team. It is important to remember that the psychological management of children with IDDM is as it would be with healthy children, but with a consideration of the special social, medical and family contexts in which these children function.

\section{Urgent and routine referrals}

Being able to respond quickly and see an urgent referral within 24 hours is important in liaison psychiatry (Black \& Cottrell, 1993). Although sometimes it is better to wait until the immediate social or family crisis has partially resolved, there are occasions when it is necessary to see a child with IDDM on the ward the same day.

It should be negotiated with the diabetes team whether routine referrals are seen by the child psychiatrist, or by other members from the psychiatrist's multi-disciplinary team, and whether the referrals receive any priority in relation to the mental health team's waiting list.

\section{Education groups}

Proactive and preventive health roles for the child psychiatrist can be combined by participating in discussion and/or education groups. Typically, separate parallel groups are run once or twice a year for the children and their parents (Watson \& Court, 1994). This does not constitute group therapy, but many of the elements of the planning and structuring of therapy groups can be applied. The groups seem to work best when the parents of children roughly the same age are in the same group (e.g. age bands $0-6$ years, $7-10$ years and 11-14 years).

Group meetings last about one and a half hours. The children usually undertake activities and games 
with an underlying health message. A useful discussion format for the parents is to base discussion around prepared vignettes on common problems they might be experiencing. For instance, for parents of very young children, a structured discussion might be led about pre-school children's food fads and how to deal with them in the context of IDDM. Games and practical exercises can be introduced into the discussion as well. Evaluations of the groups by both the children and parents is usually good. They are felt to be both informative and enjoyable, providing a good opportunity to learn from others in a similar situation.

\section{Consultation to the diabetes team}

Group therapy skills are also invaluable, in the setting of consultation, to a team. It is clearly not costeffective for a child psychiatrist to sit in the diabetes clinic every week. Consultation offers an effective and time-efficient model that allows the diabetes team to discuss difficult cases on a regular basis and to bring general management problems for discussion.

Setting-up consultation sessions requires negotiation with all the paediatric team, to find a suitable time, day and venue. The out-patient clinic is not a good venue, as it will probably be noisy and there will be interruptions from other staff. There are some common-sense practicalities. The room should be quiet, preferably comfortable and it is a good idea to provide tea and coffee. Allow 1-1.5 hours per session. Sessions should be at least monthly to retain continuity. It may be useful before the first consultation session with the diabetes team to learn more about both the way the team works, and about IDDM, by sitting in on diabetes clinics and / or accompanying the diabetes nurses on some visits to refresh the memory on diabetic management and jargon (see Box 2). In this way medical credibility can be improved among staff and families alike and can increase child psychiatrists' self-confidence about discussing IDDM with children and families.

\section{Staff support}

Staff support can be provided informally in the clinic setting or implicitly during consultation. It is helpful to team relations, and may diffuse team anxiety, if members of the diabetes team are allowed to phone the child psychiatrist directly to discuss particularly difficult or urgent problems. Boundaries can be made round this arrangement, such as the criteria for ringing or the times that are appropriate during the day. More formally it is a good idea to encourage and facilitate the setting-up of a staff support group. This may not necessarily be a longterm commitment; it may in time be appropriately run by the team staff alone.
Box 2. What a child psychiatrist should know about IDDM

What an insulin pen looks like and how it works

Formulations of insulin mixtures

What foods are included on a 'swap' carbohydrate list

Types of blood glucose meters

Accepted range of $\mathrm{HbA}_{1 \mathrm{c}}$ levels

The paediatric team's advice on frequency of blood testing

The paediatric team's acceptable $\mathrm{HbA}_{1 \mathrm{c}}$ level

\section{Useful psychological models in management}

Although psychological assessment and treatment of children with IDDM are essentially the same in principle as for any child, some psychological models are particularly useful in conceptualising and formulating a therapy plan, either when seeing an individual or in a consultation session. Box 3 gives a list of useful models with references relating to the use of these models in children with chronic diseases. Evidence-based evaluation of interventions for children with any chronic disease is very limited (Wallander \& Varni, 1998) and especially so for children with IDDM. Among the studies conducted, social skills training, education programmes and focused family therapy have all been noted to produce improved psychosocial functioning. However, none of these studies related to IDDM in children or adolescents. For this reason I will not discuss any one treatment modality in depth. Rather, several useful psychological models (Box 3 ) are discussed, and a case vignette given.

Box 3. Useful models (secondary sources related to childhood chronic disease) in formulating the problem

Transactional analysis (Brown, 1996)

Hierarchy of needs (Coles, 1996)

Grieving process (Coles, 1996)

Stress and coping model (Wallander \& Varni, 1998)

Model of behaviour change (Litt \& Cuskey, 1980) 


\section{Transactional analysis}

Transactional analysis holds that parent, adult and child ego states are present in each individual. There is conflict between these states, and we act at different levels in different situations. For example, when we are frightened or ill we revert to the child state and respond to health staff as parental figures. The hierarchy of needs' model proposes that human needs can be arranged in a hierarchy, with basic physiological needs being addressed first, then emotional, social and intellectual needs (addressed in that order).

\section{Grieving process}

The grieving process is a normal psychological adjustment to any event which can be construed as a loss. Although bereavement may be as the result of the death of a loved one, it may also follow the removal of an article, personal status or health status. For parents, a child developing IDDM can effectively be the loss of the child as the parents know it; they have to mourn this loss, and then get to know the 'new' child who has IDDM.

\section{Stress and coping model}

This model postulates disease parameters, functional dependence in daily living activities and psychosocial stressors as risk factors for psychological problems. Intrapersonal factors, social and family support and ability to process stress are seen as possible resistance factors.

\section{Model of behaviour change}

Prochaska (1996) describes a cyclical model based on cognitions about changing behaviour, through pre-contemplation (of new desired behaviour), contemplation (of changing behaviour), action (carrying out desired behaviour) and maintenance.

\section{Vignette}

The following case vignette describes a typical case which might be referred either for direct help or for consultation.

Sarah was a seven-year-old girl who lived with her parents and older brother. She developed IDDM at the age of three following a prolonged viral infection. She had a blood sugar of $25 \mathrm{mmol} / 1$ on admission, but was quickly stabilised and only remained in hospital overnight because her mother said that she could not cope with her at home immediately. Her mother, Viv, was very upset at the time of diagnosis; she had suspected that Sarah had IDDM and had taken her to their general practioner (GP) who initially just thought she had gastro-enteritis. Viv still feels very angry with the GP about this and avoids going to see him. Sarah's father, Steve, withdrew from learning about how to give injections and how to monitor Sarah's blood sugar levels and did not play an active role in looking after her until six months after diagnosis.

Sarah was well-controlled on two insulin injections a day for the first year, despite being very difficult with her mother about her diet. She refused to eat breakfast at all, although prior to diagnosis she had always eaten a bowl of cereal and a slice of toast. Viv would offer her whatever was in the kitchen cupboard and she would say that she did not like anything except chocolate biscuits. Viv on one occasion phoned the GP and asked him to come round to see how terrible things were, and the diabetes nurse specialist described a home visit were she had found Viv crying hysterically on the sofa while Sarah was happily eating chocolate biscuits in front of her. At this time, Sarah's $\mathrm{HbA}_{1 \mathrm{c}}$ was $6.5 \%$.

In the next two years however, her diabetic control deteriorated (blood glucose between 2 and $18 \mathrm{mmol} /$ 1 most days) and her last $\mathrm{HbA}_{1 \mathrm{c}}$ was $12 \%$. Consultant paediatricians aim to keep childrens' $\mathrm{HbA}_{1 \mathrm{c}}$ values below $10 \%$. The diabetes nurse specialist visited the family fortnightly over a two-month period and worked on a practical programme of a healthy diet, exercise and twice-daily blood sugar monitoring which was negotiated with Sarah and her parents. Viv told the diabetes nurse specialist that she doubted that the programme would work as Sarah would sabotage it from the outset. Sarah's blood glucose levels stabilised slightly but then deteriorated again and she was admitted to the paediatric ward with moderate diabetic ketoacidosis (blood glucose $25 \mathrm{mmol} / \mathrm{l}$, blood $\mathrm{pH} 7.2$ and blood bicarbonate $12.5 \mathrm{mmol} / \mathrm{l})$. When Sarah was medically fit, the diabetes nurse specialist agreed with the family for a referral to the team child psychiatrist to be made.

At assessment, all four of the family were present. Steve described the trauma of being made redundant a year after Sarah developed IDDM and had recently started up his own computer business. Viv was very angry and close to tears throughout the assessment and stated that she would never have had Sarah had she known that she would develop IDDM and if she became pregnant again, she would abort the child because she could not possibly look after another child with IDDM. Much of the interview was spent talking about Viv. She had felt very depressed after the birth of both children but had not sought help because she came from a family who believed in 'pulling yourself together'. She denied feeling depressed at present but the child psychiatrist had the impression that Viv was reluctant to talk in front of her husband. Sarah looked detached from much of the discussion and only became animated when talking about her ballet class, where she was achieving very highly. At the end of the initial assessment session the psychiatrist was surprised by how many 
gaps there were in the assessment, particularly facts relating to Sarah: most of the information gathered had related to Viv and Steve.

\section{References}

Baum, J. D. (1990) Children with diabetes. British Medical Journal, 301, 502-503.

Black, D. \& Cottrell, D. (1993) Seminars in Child and Adolescent Psychiatry. London: Gaskell.

Brown, F. (1996) Counselling for patient empowerment. Practical Diabetes International, 13, 189-190.

Cadman, D., Boyle, M. H., Offord, D. R., et al (1986). Chronic illness and functional limitation in Ontario children: findings of the Ontario Child Health Study. Canadian Medical Association Journal, 135, 761-767.

Coles, C. (1996) Psychology in diabetes care. Practical Diabetes International, 13, 55-57.

Court, S., Sein, E., McCowen, C., et al (1988) Children with diabetes mellitus: perception of their behavioural problems by parents and teachers. Early Human Development, 16, 245-252.

Diabetes Control and Complications Trial Research Group (1993) The effect of intensive treatment of diabetes on the development and progression of long-term complications in insulin-dependent diabetes mellitus. New England Journal of Medicine, 329, 977-986.

Kovacs, M., Iyengar, S., Goldston, D., et al (1990) Psychological functioning among mothers of children with insulin-dependent diabetes mellitus: a longitudinal study. Journal of Consulting and Clinical Psychology, 58, 189-195.

Lavigne, J. V. \& Faier-Routman, J. (1992) Psychological adjustment to pediatric physical disorders: a meta-analytic review. Journal of Pediatric Psychology, 17, 133-157.

Litt, F. \& Cuskey, W. R. (1980) Compliance with medical regimens during adolescence. Pediatric Clinics of North America, 27, 3-15.

Magrath, G., Hartland, B. V. \& Nutrition Subcommittee of the British Diabetic Association's Professional Advisory Committee (1993) Dietary recommendations for children and adolescents with diabetes: an implementation paper. Diabetic Medicine, 10, 874-885.

Metcalfe, M. A. \& Baum, J. D. (1991) Incidence of insulin dependent diabetes in children aged under 15 years in the British Isles during 1988. British Medical Journal, 302, 443447.

Prochaska, J. O. (1996) A stage paradigm for integrating clinical and public health approaches to smoking cessation. Addictive Behaviours, 21, 721-732.

Shield, J. P. H. \& Baum, J. D. (1994) Prevention of long-term complications in diabetes. Archives of Disease in Childhood, 70, 258-259.

Wallander, J. L. \& Varni, J. W. (1998) Effects of pediatric chronic physical disorders on child and family adjustment. Journal of Child Psychology and Psychiatry, 39, 29-46.

Watson, C. \& Court, S. (1994) Group education in the diabetes clinic using a games format. Practical Diabetes, 11, 142-144.

Worrall, A. (1996) Psychological Aspects of Childhood Diabetes. MD thesis, University of Leeds.

\section{Multiple choice questions}

1. Sarah apparently had good glycaemic control (glycated haemoglobin of $6.5 \%$ ) while still eating chocolate biscuits for breakfast:

a therefore her mother must have been recording false blood sugar readings b this is possible because glycated haemoglobin only gives an average measure of control

c Sarah must have been eating little else but chocolate biscuits

d therefore there is not a problem with Sarah's diet.

2. The following therapeutic options might be useful in thinking about Viv's distress:
a bereavement counselling
b transactional analysis
c fluoxetine alone
d fluoxetine and chlorpromazine.

3. The following would be reasonable formulations of the problem:

a Sarah is a disturbed child and requires intensive individual therapy

b Viv and Sarah have a difficult relationship and this may be related to Viv's own experience of parenting

c Viv and Steve have divergent roles in the care of Sarah and this is a healthy approach to the care of a child with a chronic disease

d Sarah receives considerable secondary gain from the negative attention she receives by refusing food; a behavioural programme could resolve this.

4. In the stress and coping model, important risk factors for Sarah becoming psychologically disturbed would be:

a her father's redundancy

b her high $\mathrm{HbA}_{1 \mathrm{c}}$ level

c becoming acidotic

d her regular attendance at ballet class.

5. In the stress and coping model, important resistance factors to Sarah becoming psychologically disturbed are:

a her father starting a new job

b her high $\mathrm{HbA}_{1 \mathrm{c}}$ level

c admission to hospital for diabetic ketoacidosis

d her regular attendance at ballet class.

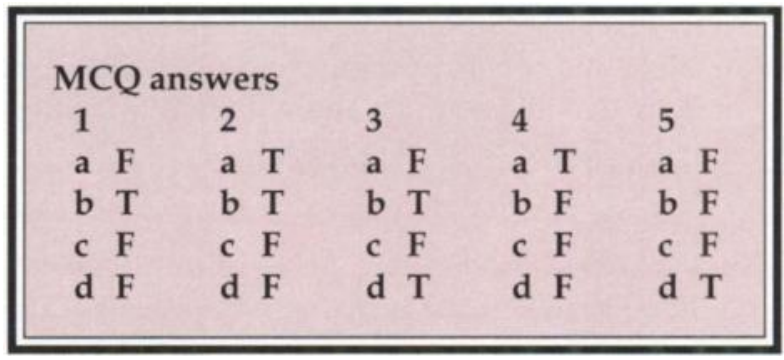

